\title{
Mutational spectrum and genotype-phenotype relationships in a cohort of Romanian hereditary angioedema patients caused by $\mathrm{C} 1$ inhibitor deficiency
}

\author{
Gabriella Gábos ${ }^{1,2}$, Dumitru Moldovan²,3, Daniela Dobru, ${ }^{4,5}$, Enikő Mihály ${ }^{2,3}$, \\ Noémi Bara ${ }^{2}$, Valentin Nădășan ${ }^{2,6}$, Adina Hutanu ${ }^{7}$, Katalin Csép ${ }^{8 *}$
}

\section{Lotus Life Clinic, Târgu Mureș, Romania}

2. Romanian Network for Hereditary Angioedema, Romania

3. Mures County Hospital Allergology-Immunology Department, Târgu Mureș, Romania

4. University of Medicine, Pharmacy, Sciences and Technology of Târgu Mureș, Romania

5. Mures County Hospital Gastroenterology Department. Târgu Mureș, Romania

6. Department of Hygiene, University of Medicine, Pharmacy, Sciences and Technology of Târgu Mures, Romania

7. Center for Advanced Medical and Pharmaceutical Research, University of Medicine, Pharmacy, Sciences and Technology of Târgu Mureș, Romania

8. Genetics Department, University of Medicine, Pharmacy, Sciences and Technology of Târgu Mureș, Romania

\begin{abstract}
Background: Hereditary angioedema due to C1 inhibitor deficiency (C1-INH-HAE) caused by SERPING1 mutations is a rare monogenic disorder characterized by a high frequency of de novo mutations, allelic heterogeneity and populational differences. Geno- and phenotype correlation data are limited. Addressing the pathogenic complexity, we proposed to analyze the clinical and genetic characteristics in a set of Romanian patients. Material and Methods: 49 patients from 22 unrelated families with C1-INH-HAE were investigated, by calculating clinical severity score (CSS), C1-INH and C4 level assessment by nephelometric assays, C1-INH function study by functional enzyme-linked immunosorbent assay, and mutation analysis by sequencing and MLPA. Clinical manifestations by missense vs other mutation mechanisms were compared. Results: The mean age at diagnosis and onset was $28.8 \pm 14.7$ and $15.1 \pm 15.2$ years, while the diagnostic delay $13.1 \pm 10.1$ years. CSS ranged from 2 to 9 , with a mean of 5.4 1.8. The frequency of missense and nonsense mutations, splice defects, frameshift mutations and large gene rearrangements was 61.22, 6.12, 22.4, 6.12 and 4.08\%; in the regulatory sequence no mutation was described. In type II, only missense mutations were noted. Lower levels of C1-INH characterized index cases caused by mechanisms other than missense mutation, with more severe consequences on protein synthesis $(p=0.017) .53 \%$ of the
\end{abstract}

\footnotetext{
*Corresponding author: Katalin Csép, Genetics Department, University of Medicine, Pharmacy, Sciences and Technology of Târgu Mureș, Târgu Mureș, Romania. E-mail: genetica.umftgmro@gmail.com
} 
cases were identified by familial screening. Conclusion: A later onset of disease manifestations and a higher frequency of missense mutations characterize HAE in Romanian patients with SERPING1 mutation. Genetic analysis improves the management of affected families, and may inform about disease severity.

Keywords: hereditary angioedema, C1 inhibitor deficiency, SERPING1

Received: 13 ${ }^{\text {rd }}$ February 2019; Accepted: 31 st May 2019; Published: $18^{\text {th }}$ June 2019

\section{Introduction}

Hereditary angioedema due to $\mathrm{C} 1$ inhibitor deficiency (C1-INH-HAE) is a rare monogenic disorder with autosomal dominant inheritance and an estimated prevalence of 1:10,000-50,000 in the general population. (1) Insufficient levels of functional complement component 1 inhibitor $(\mathrm{C} 1-\mathrm{INH})$ lead to excessive levels of bradykinin, the main mediator of angioedema, thus the clinical picture is characterized by episodes of self-limiting, localized edema due to an increased vascular permeability, affecting different body sites, for instance, limbs, face, intestinal tract or airways. If not treated properly, laryngeal edema is associated with an extremely high risk of asphyxiation, and, consequently, death.(2)

The disorder is caused by various mutations affecting the C1-INH gene - SERPING1 (OMIM*606860, C1I; C1INH SERPIN PEPTIDASE INHIBITOR). $(3,4)$ HAE type I develops when C1-INH levels are reduced (85\%), and type II in case of a dysfunctional C1-INH (15\%). (5) The group with normal C1-INH (HAE-nC1INH) comprises: HAE with mutation in F12 gene (HAE-FXII), HAE with mutation in the angiopoetin-1 gene (HAE-ANGPTI), HAE with mutation in the plasminogen gene (HAE-PLG), and HAE due to unknown mutations (HAEUNK).(5)

The SERPING1 gene is approximately $17 \mathrm{~kb}$ long, comprises 8 exons and 7 introns, and it is located in 11q12.1. It presents an unusual promoter with no TATA sequence but a TdT-like initiator and polypurine-polypyrimidine tract.(4) More than 450 distinct SERPING1 mutations as- sociated with C1-INH HAE have been described to date, produced by various mechanisms, ranging from the most frequent point mutations causing missense, nonsense, splice site or frameshift mutations, to small and large insertions or deletions (HAEdb, http://www.hae.enzim.hu). (6) Allelic heterogeneity and the high frequency of de novo mutations in $20-25 \%$ of the cases are due to mutagenic liability.(6)

Genetic complexity poses difficulties in patient management, from diagnosis and prognosis assessment to the choice of optimal treatment. Ethnic differences have been shown to be important, and may influence disease manifestations, severity and progression.(7) Studies analyzing genoand phenotype correlations with potential predictive value are limited, and the results are controversial.(7-11) Based on these considerations, we proposed to analyze the clinical and genetic characteristics in a set of patients, intended for a more tailored management of C1-INH-HAE affected Romanian families.

\section{Material and Methods}

Forty-nine patients from 22 unrelated families with C1-INH-HAE were included in the study, recruited through the Romanian Registry of Hereditary Angioedema (www.haenet.ro). Diagnosis of C1-INH-HAE was based on family and/or personal history of recurrent angioedema without urticaria associated with antigenic and/or functional C1-INH deficiency. Serum concentrations of C1-INH and C4 were quantified using nephelometric assays (BN Prospec, Siemens Healthcare $\mathrm{GmbH}$ ), while functional $\mathrm{C} 1-\mathrm{INH}$ was 
measured by functional enzyme-linked immunosorbent assay (MicroVue functional C1-inhibitor, Quidel Corporation) on automated ELISA analyser (Adaltis, Italy). Clinical severity score was calculated using the age of onset, number of organs affected and long-term prophylaxis, and expressed from 0 to 10 , as proposed by Bygum et al.(8) Peripheral venous blood samples were collected from 28 patients, 22 index cases and 6 first degree relatives exhibiting the disease, and sent to the Department of Immunology \& Histocompatibility School of Medicine, University of Thessaly for genetic analysis. Genomic DNA was isolated, and SERPING1 mutations were detected by sequencing after PCR amplification of all exons and exon/intron boundaries, followed by long-range PCR and MLPA for the identification of large rearrangements in 28 cases, as published previously.(7) In symptomatic first degree relatives with a clinical diagnosis but no molecular analysis of index cases with a known mutation, the presence of the same mutation was considered.

The study was carried out in accordance with the Principles of the Declaration of Helsinki and a written informed consent was obtained from the patients.

Results were expressed as mean, standard deviation, and percentage. To test genotype - phenotype associations on the index cases sample, the Chi-square and Mann-Whitney tests were applied for categorical and continuous variables, respectively. Genotype-phenotype associations in the extended sample including family members were explored using the complex sample Chi-square test and complex sample student $t$ test for categorical and continuous variables respectively, with controlling for the intracluster correlations due to patient aggregation at family level in SPSS v. 22.0.(12,13) A two-tailed p-value of less than 0.05 was considered statistically significant.

\section{Results}

Twenty-two index cases and a total of 49 patients from the twenty two unrelated families with HAE type I and II were included in the study. The study population included 25 female and 24 male subjects from 22 unrelated families, 46 adults and 3 patients under 18 years. The majority of patients $(81.63 \%)$ had type I, while $18.37 \%$ type II of HAE. The mean age at the onset of clinical symptoms was $15.1 \pm 15.2$ years, ranging from 0 to 70 years, and the mean age at diagnosis was $28.8 \pm 14.7$ (from 3 to 70 years), with a diagnostic delay of $13.1 \pm 10.1$ years (up to 38 years in one of the cases). $10.20 \%$ of symptomatic patients were receiving long-term prophylaxis with attenuated androgens (Danazol), but none of them used antifibrinolytic agents or C1-INH for long-term prophylaxis. $89.8 \%$ of the patients had no treatment at all. Major symptoms were abdominal edema in 44 patients $(89.79 \%)$, followed by skin edmea in 35 patients $(71.42 \%)$, laryngeal edema in $46.93 \%$, and facial edema in $44.89 \%$. Family history of HAE was present in $87.8 \%$ (43/49); six patients $(12.2 \%)$ had no known cases in the family.

Clinical and genetic characterization of the study group is presented in Table I.

Over half of the cases were caused by missense mutations, while approximately a quarter by splice defects; other point mutations and large rearrangements were found relatively rare.

Thirty missense mutations were identified in thirteen C1-INH-HAE families: three in exon 3, c. $550 \mathrm{G}>\mathrm{A}$, c. $498 \mathrm{C}>\mathrm{A}$ and c. $503 \mathrm{C}>\mathrm{A}$; four in exon 7, c. $1193 \mathrm{~T}>\mathrm{C}$, c. $1187 \mathrm{~T}>\mathrm{C}, \mathrm{c} .1154 \mathrm{~A}>\mathrm{G}$, c. $1223 \mathrm{~A}>\mathrm{G}$; four in exon 8 , c. $1396 \mathrm{C}>\mathrm{T}$, c. $1418 \mathrm{~T}>\mathrm{G}, \mathrm{c} .1396 \mathrm{C}>\mathrm{T}$ and c. $1397 \mathrm{G}>\mathrm{A}$; one in exon 6, c.988T $>\mathrm{G}$ and one in exon 5, c.860T $>\mathrm{C}$. Three nonsense mutations were described in three C1-INH-HAE families: one in exon 3 , c. $124 \mathrm{G}>\mathrm{T}$ and two in exon 7, c. $1042 \mathrm{C}>\mathrm{T}$. Three frameshift mutations were identified in 


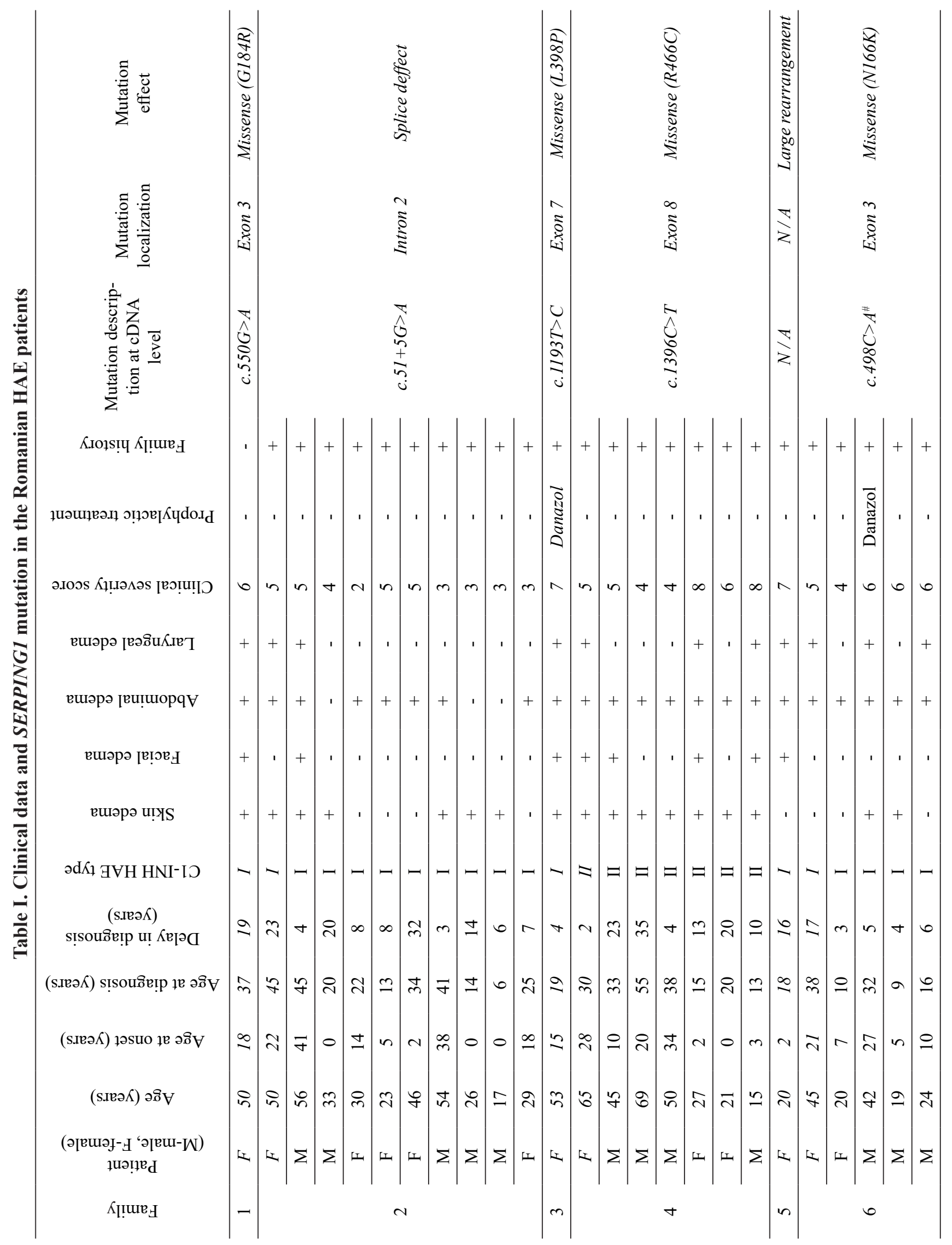




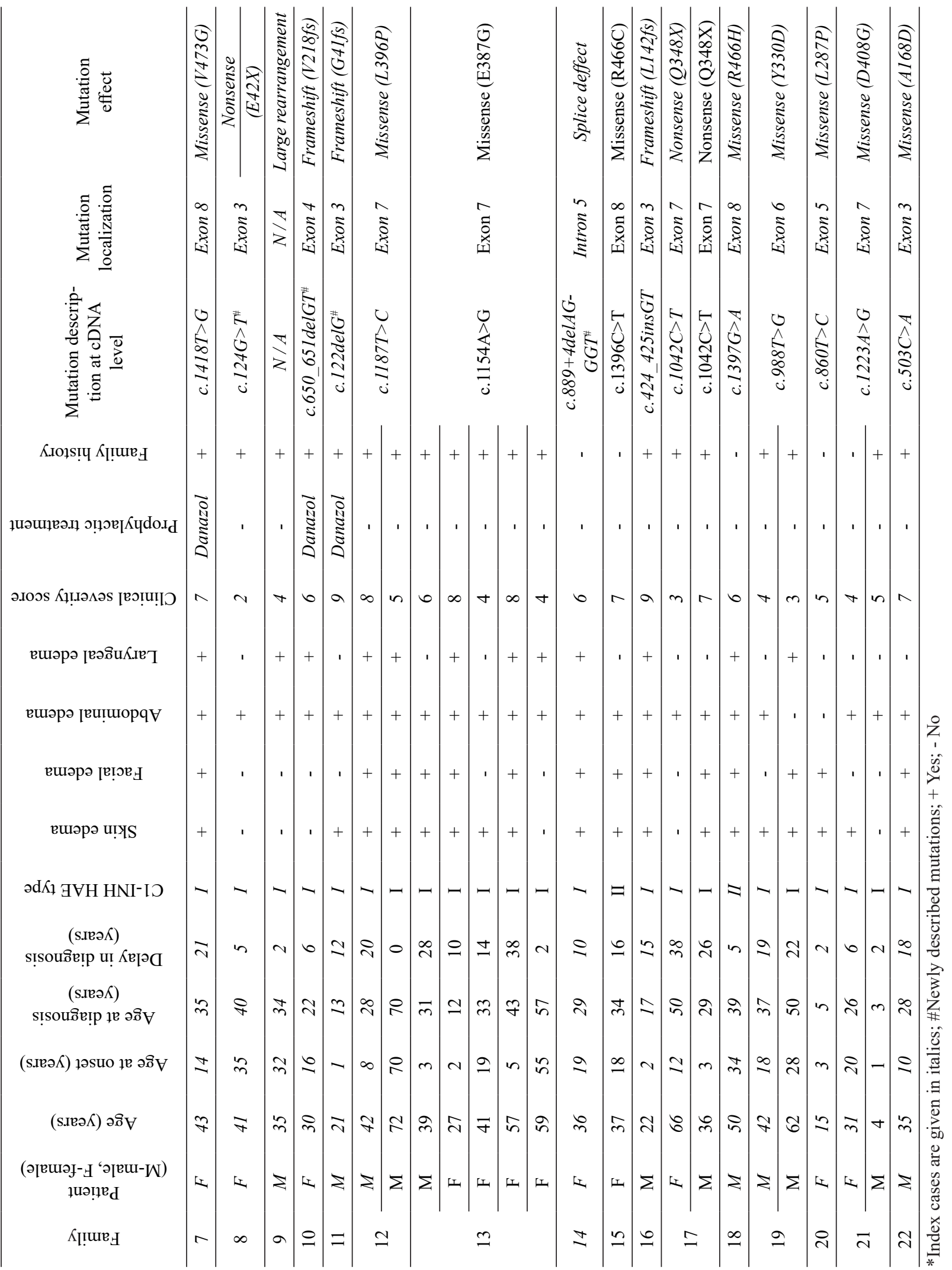


three C1-INH-HAE families: one in exon 4, c.650_651delGT, and two in exon 3, c.122delG and c.424_425insGT. In our cohort of patients, 11 splicing defects were identified, c. $51+\mathrm{fG}>\mathrm{A}$ in intron 2 and c.889+4delAGGGT in intron 5 in two families with C1-INH-HAE type I.

Distribution of mutation mechanisms as well as localization and type of mutations in the SERPING1 gene of Romanian C1-INH-HAE patients are presented in Figure 1 and 2.
To address possible geno- and phenotype correlations, clinical data was compared according to the type of mutation, patients being classified in two groups based on the presence of missense mutations (group 1), or any other mutation mechanisms (group 2), as seen in Table II. A tendency of less severe clinical manifestations was noted dependent on the mutation mechanism, in the presence of missense mutations leading to the substitution of a single aminoacid as compared

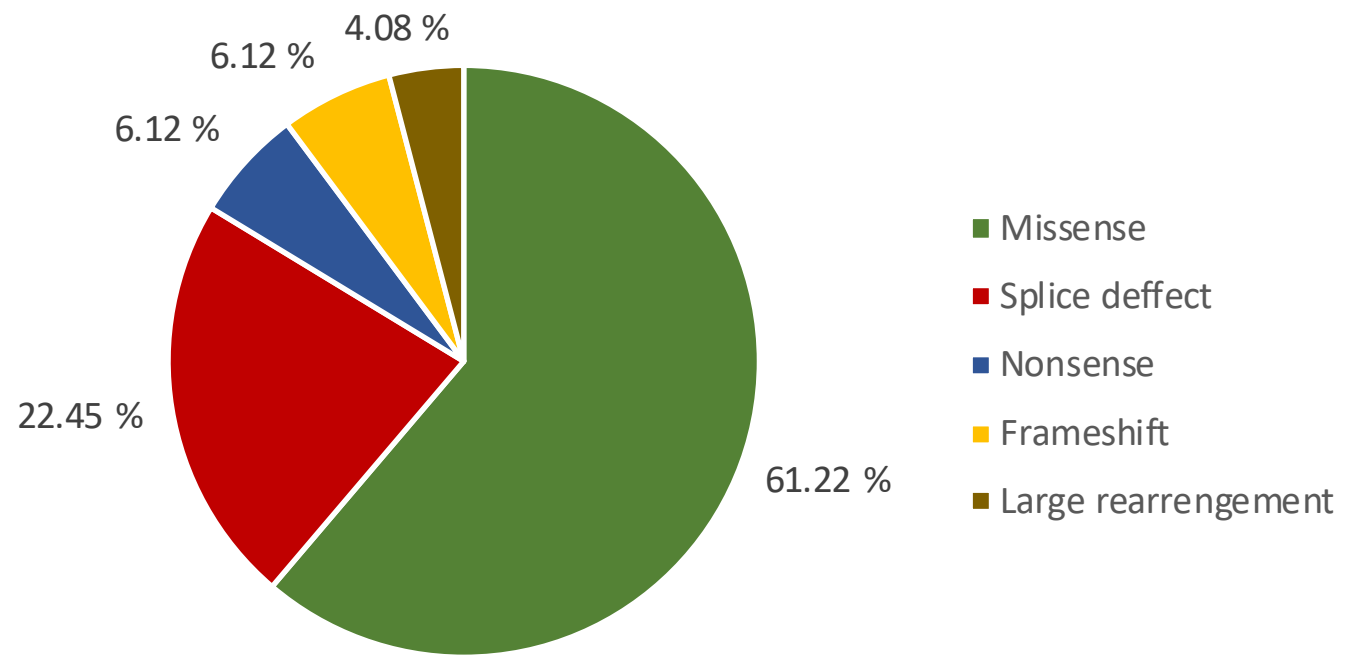

Fig. 1. Distribution of the SERPING1 mutation mechanisms in Romanian HAE patients

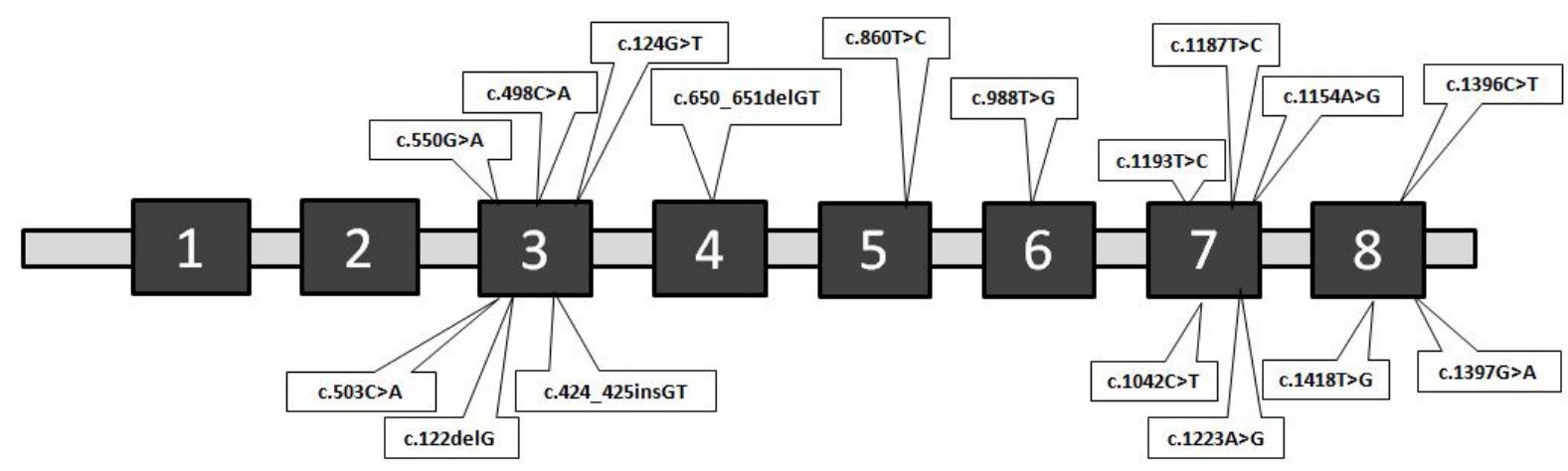

Fig. 2. Localization and types of mutation in the SERPING1 gene of Romanian C1-INH-HAE patients 


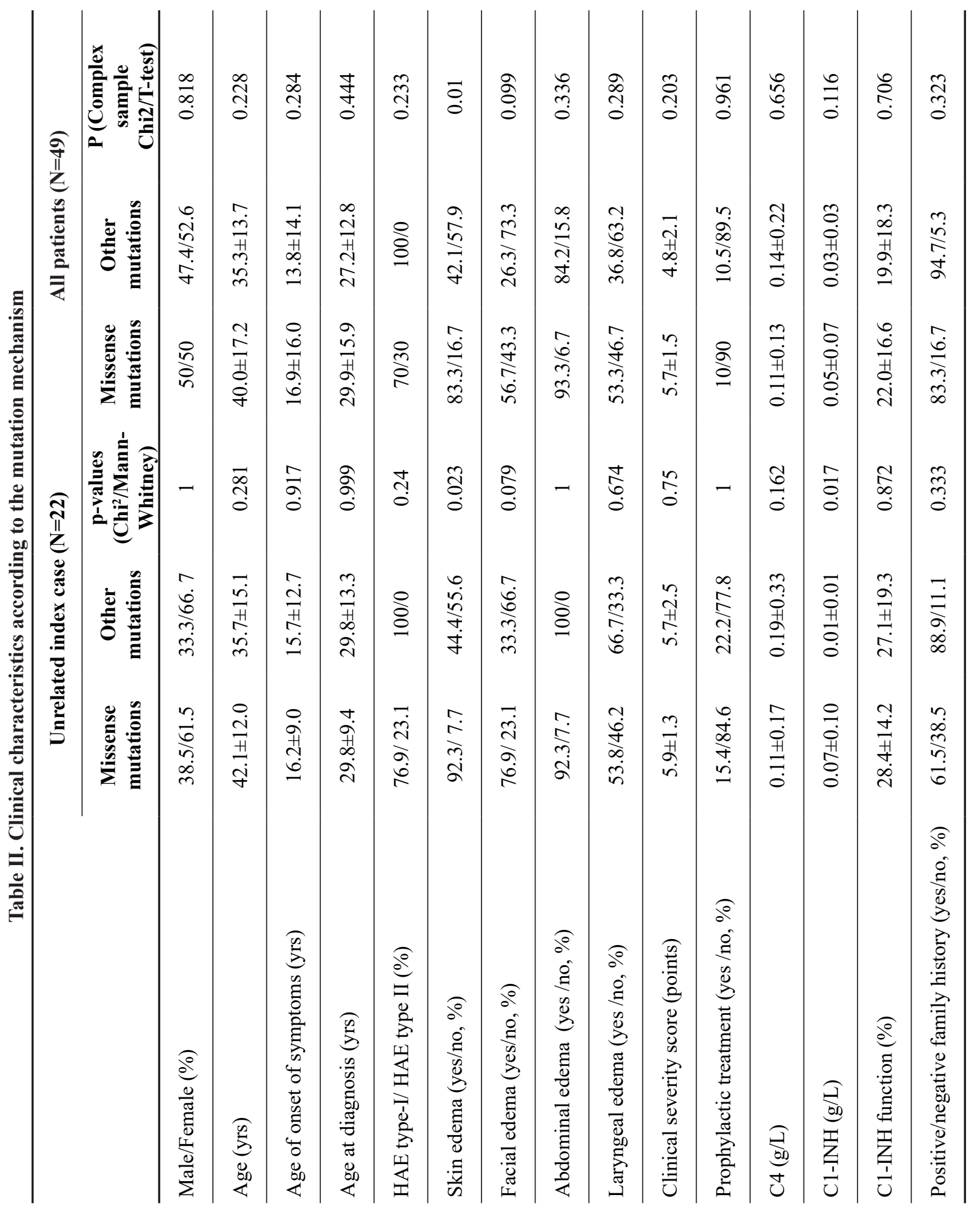


with mutations causing more severe changes in the protein structure.

Autosomal dominant inheritance pattern was characteristic, with variation of the age at the onset of the disease within the family suggesting variable clinical expressivity, as shown in a representative case (Figure 3).

\section{Discussion}

In an effort to further our understanding of the disorder and improve patient management, the Romanian Registry Hereditary Angioedema (RRHAE) was established in 2010, inspired by the European Hereditary Angioedema Registry. Today it includes 108 HAE type 1 and 2 patients belonging to 45 unrelated families. With 19,5 million inhabitants and calculated with a prevalence of 1:50,000, there are an estimated 490 patients in Romania, suggesting that approximately 400 cases are still undiagnosed. The ratio of types I and II of the disease appears similar to other countries ( 81.63 vs $18.37 \%$ of $\mathrm{C} 1-\mathrm{INH}-$ HAE type I and II).(14-16)

The mean age at diagnosis and onset was $28.8 \pm 14.7$ and $15.1 \pm 15.2$ years, while the diagnostic delay $13.1 \pm 10.1$ years, comparable to other European countries (i.e., 12 years in France and 16.3 years in Denmark).(14,15) Clinical severity score based on the age of onset, organs affected, and long-term prophylaxis ranged from 2 to 9 , with a mean of $5.4 \pm 1.8$. Long-term prophylaxis with attenuated androgens (Danazol) was administered to only $10.20 \%$ of the patients, significantly lower than in other European countries, including those in neighboring countries from the region. $(9,10)$ The later age of onset previously reported in a regional study including 14 Romanian patients,(7) on this larger set is not confirmed, and in this cohort of 49 patients, the mean age of onset was found similar to other populations.(14-16)

Despite simple monogenic autosomal dominant inheritance, genetic complexity characterizes

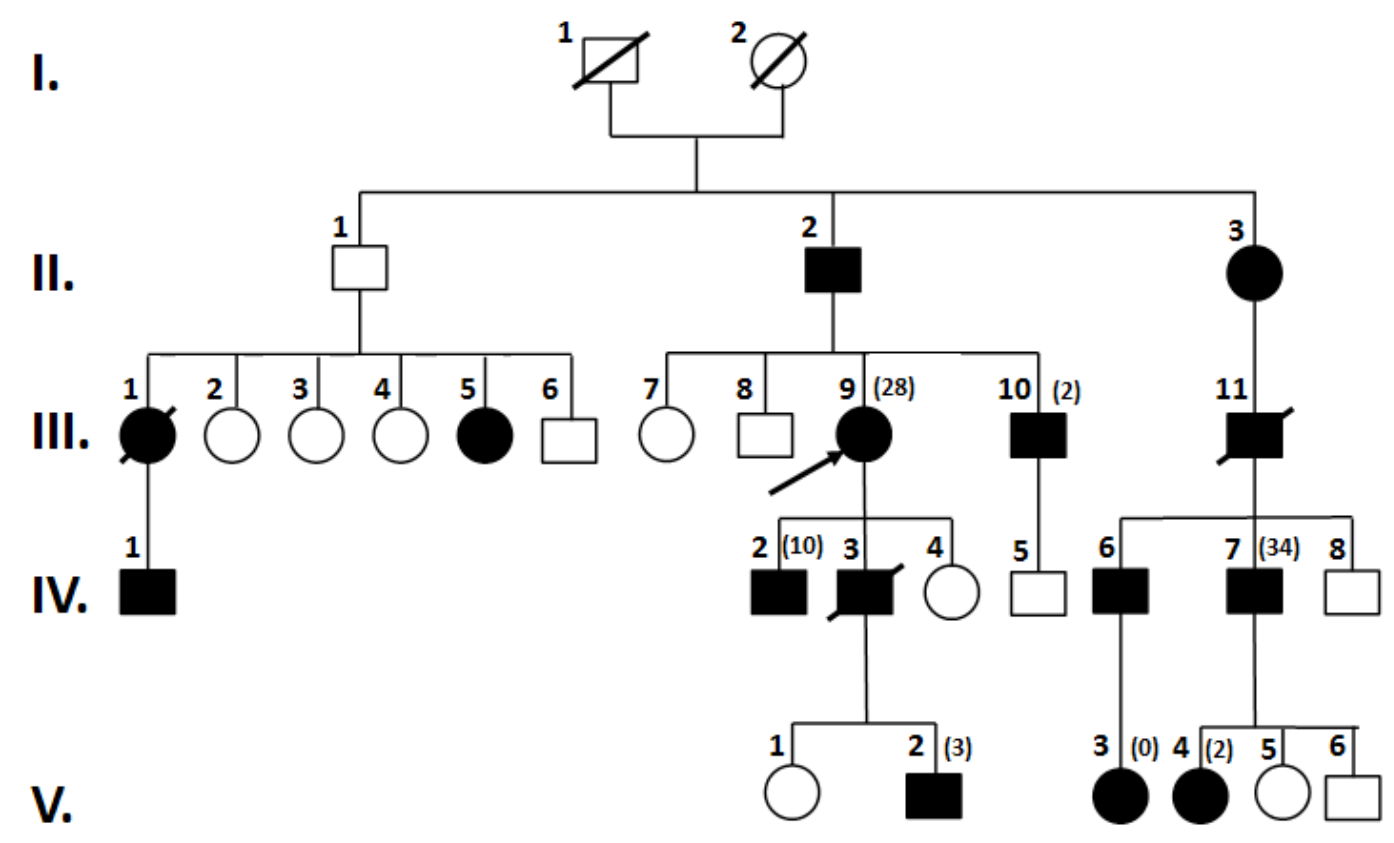

Fig. 3. Medical family tree in a Romanian family with HAE-type II, caused by a missense SERPING1 mutation (c.1396C $>$ T, R466C). Age of onset is shown between brackets. 
HAE, and may make counseling difficult. Due to heterogeneity, inconsistent genotype-phenotype relationships, mutation analysis is not mandatory for establishing the diagnosis. Both locus and allele heterogeneity are possible: most often variable mutations in the SERPING1 gene cause the clinical manifestations, but in $25 \%$ of the cases $F 12$ gene is altered, or other mutations may be involved. In our patients no such case was identified, but the $-46 \mathrm{C} / \mathrm{T}$ polymorphism in $F 12$, a confirmed modifier of HAE phenotype was investigated in a subset of 14 type I and II patients, and found in $17.5 \%$, similar to other countries in the region.(17)

C1-INH-HAE diagnosis is based on the measurement of antigenic and/or functional C1-INH plasma levels, but the optimal management of patients and their families includes genetic testing. Mutation analysis may contribute with valuable additional information allowing familial screening, pre-symptomatic, prenatal or pre-implantation diagnosis, confirmation of casual variants in apparently healthy mutation carriers, or confirmation of diagnosis when classical approaches are limited (i.e. patients less than 1 year old), while consistent geno- and phenotype relationships, if identified, could offer clues about prognosis. Beyond the scientific value, mutation analysis of our patients significantly helped the management of affected families, by confirmation of diagnosis and identification of mutation carrier relatives, $53 \%$ of our cases having been identified by familial screening. It has been reported that $5 \%$ of adult cases are identified only after a child is diagnosed in the family, and approximately $14 \%$ of mutation carriers remain asymptomatic throughout their life, $(6,7)$ which was not the case in our patients.

Interpretation of mutation analysis is burdened by important allelic heterogeneity with over 450 mutations described so far in the SERPING1 gene. Moreover, various polymorphisms must be differentiated from causative mutations using limited approaches, like segregation analysis or functional studies. The high mutation rate is explained by the position of the gene locus situated close to the centromere, in a region with high Alu repeat content and $\mathrm{CpG}$ sites.(18,19) In our investigated cohort, $87.75 \%$ were familial and $12.25 \%$ appeared as sporadic cases, suggesting de novo mutations. Family history was positive in approximately $2 / 3$ of the unrelated index cases, comparable to $20-25 \%$ described in the literature.(20) The high frequency of newly described mutations makes simple analytical methods less applicable and the use of conventional investigational approaches is considered to be fraught with pitfalls (e.g. identification of novel missense mutations may prevent the description of indel mutations leading to truncated proteins or deep intronic mutations affecting splicing). $(6,7,21)$ Thus sequencing is necessary, which usually targets exon sequences and exon-intron boundaries, and is combined with long-range PCR and MLPA (multiplex ligation-dependent probe amplification) for the assessment of large rearrangements, as it was done also in our patients.(7) Recent analytical developments may further improve existing diagnostic possibilities. Targeted next-generation sequencing can provide the necessary analytical sensitivity, and is currently recommended for implementation as a first line approach for genetic testing, or as a confirmatory technique.(21)

Important ethnic differences have been noticed regarding the mutation mechanisms.(7) Epigenetic influences and regional environmental factors like diet or radiation are quoted to be responsible for the mutation profile different in various countries.(7) Compared with recent publications and current SERPING1 mutation database data, the mutation mechanism in Romanian patients shows an unusual distribution, $(7,10,22,23)$ confirming previous reports now on a larger group of cases, with a significantly greater proportion of missense mutations and splice defects $(61.22 \%$ and 
$22.4 \%$, respectively), a lower frequency of nonsense and frameshift mutations, as well as large gene rearrangements than in other populations; no mutations were described in the regulatory sequence. Novel mutations described in Romanian patients include c.122delG, c.650_651delGT (frameshift mutations), c. 124G $>$ T (nonsense mutation), c.498C $>$ A - N166K (missense mutation), c.889+4delAGGGT (splice defect), as published previously.(7)

Mutation localization in control sequences, exons or introns may perturb the production of the gene product, cause an abnormal aminoacid sequence, protein folding, and thus alter $\mathrm{C} 1-\mathrm{INH}$ function. Leading to an unusually high mutation rate, the $17 \mathrm{Alu}$ sequences situated in introns (especially intron 4 and 6) predispose to non-homologous recombination with the appearance of large rearrangements - deletions and duplications, while the $\mathrm{CpG}$ sites predispose to substitutions by spontaneous deamination (especially in codon 444, or 466 according to HUGO numbering).(6,7)

Though mutations are distributed all over the gene, certain mutational hot-spots were identified with nucleotide sequences critical for protein structure and function, like exon 8 encoding hinge regions and the reactive center of C1-INH. (24-28) 20.4, 2.04, and 2.04\% of the Romanian cases presented mutations in exons 8,5 and 6 , in total $24.48 \%$ in the locations considered the most frequent in the cited literature.

Genetic heterogeneity motivates efforts to clarify genotype-phenotype relationships. If identified and confirmed, robust correlations could provide important information about prognosis, the natural course of the disease, which could assist an optimized management, a more tailored approach based on genetic information. So far, however, results of the limited observations are inconsistent.(7-10,29) Some reported little if any correlations, (6) while Speletas et al. have shown in a study including a set of 14 Romanian pa- tients that missense mutations associate with a less severe natural course, the age of onset below 10 years occurring significantly more rarely than in other mutations.(7) Surprisingly, in C1-INHHAE missense mutations almost always affect gene product level and/or function, causing clinical manifestations. It is not fully explained yet why the substitution of a single aminoacid leads almost invariably to the loss of C1-INH function, but protein misfolding and multimerization have been quoted as possible causes.(30-32) The position and type of the changed aminoacid may further explain the important variations in the clinical picture we have seen; furthermore, some mutations have also been described to have different consequences in different cell types.(33) Frameshift or nonsense mutations with profound changes in aminoacid sequence and leading to truncated proteins result in unstable gene products, leading to reduced C1-INH levels and HAE type 1 (80-85\%). Almost exclusively, missense mutations cause the less frequent type II of the disease $(15-20 \%)$, characterized by normal antigenic levels, but reduced C1-INH function (1,34-36). In $70 \%$ of the cases, these mutations are situated in exon 8 , a functionally critical region encoding the reactive site in serpins, especially Arg444 (or Arg466). Pathogenic missense mutations were found in $3 / 4$ of the unrelated Romanian index cases presenting HAE type I, and $100 \%$ of type II patients, situated exclusively in exon 8 . In heterozygous patients, the unusually markedly reduced $\mathrm{C} 1-\mathrm{INH}$ levels to $5-30 \%$ of the normal level is suspected to be related to an increased catabolic rate and reduced synthesis with the down-regulation of the normal allele, a possible dominant negative effect, though as of now it remains insufficiently explained. (19,37,38)

Inter- and intrafamilial variable clinical expressivity, as suggested also by the changeable age of onset in the represented family tree (Figure 3 ), may be related to genetic and non-genetic 
factors. Various factors like SERPING1 gene polymorphisms (e.g. rs4926, rs28362944) and mutations in other genes (e.g. $C 4 B, B D K R 1$ and 2, $M B L 2, A C E$ ) were studied as potential modifiers.(6) The $-46 \mathrm{C} / \mathrm{T}$ polymorphism in the F12 gene promoter sequence (rs1801020) appeared to correlate consistently with disease severity, as reported previously in a study also including a set of Romanian patients.(7) Given the characteristic later manifestation in Romanian patients, the presence of the G allele of the KLKB1-Ser143Asn polymorphism investigated in a subset of 19 patients was reported to appear as an independent genetic factor correlated with a delayed onset in C1-INH HAE. (39)

Based on previous observations,(7) in order to investigate genotype-phenotype associations, we separated patients in two groups carrying the frequent missense mutations with presumably less severe changes in gene product structure, and nonsense, splice, frameshift mutations, insertions and deletions of variable sizes producing a more severe effect on C1-INH level and function. Comparison of severity and clinical manifestations demonstrated that according to the presumed effect on gene product stability dependent on mutation mechanism, there was a more severe reduction of C1-INH level in carriers of other than missense mutations as also noted by other authors, (7-11) which reached the level of statistical significance, but only in probands ( $p=0.017$ vs 0.116 ). With marked variations in each group, neither the age of onset nor the calculated score denoting clinical severity demonstrated significant differences. Interpretation of our current results remains, however, limited by the relatively low number of cases, and insufficient genetic investigations, analysis of modifying genetic factors. It will be interesting to see how the currently developed global HAE registry data with more statistical power will clarify the tendencies noted in our cohort.

\section{Conclusion}

A later onset of disease manifestations and a higher frequency of missense mutations characterize HAE in Romanian patients with SER$P I N G 1$ mutation. Genetic analysis provides information necessary for the optimization of management in the affected family, assisting the clarification of diagnosis in affected relatives. Lower levels of C1-INH may characterize mutations caused by mechanisms other than the substitution of a single aminoacid. In Romanian patients with HAE type II, exclusively missense mutations were noted.

\section{Authors' contribution}

Gabriella Gábos (Conceptualization; Formal analysis; Methodology; Validation; Visualization; Writing - original draft)

Dumitru Moldovan (Data curation; Supervision; Validation; Visualization)

Daniela Dobru (Conceptualization; Formal analysis; Supervision; Validation)

Enikő Mihály (Data curation; Formal analysis; Supervision; Validation; Visualization)

Noémi Bara (Data curation; Supervision; Validation; Visualization)

Valentin Nădăan (Conceptualization; Data curation; Supervision; Validation)

Adina Hutanu (Formal analysis; Methodology; Validation; Visualization)

Katalin Csép (Conceptualization; Methodology; Writing - original draft; Writing - review \& editing; Preparation of manuscript)

\section{Acknowledgments}

Our sincere thanks go to Prof. Dr. Anastasios Germenis, head of the Department of Immunology \& Histocompatibility School of Medicine, University of Thessaly, Greece, for the genetic analysis of the blood samples. We also thank the 
patients who participated in this study and their families.

\section{Declaration of interests}

We declare no competing interests

\section{References}

1. Longhurst $\mathrm{H}$, Cicardi M. Hereditary angio-oedema. Lancet. 2012;379(9814):474-81 DOI: 10.1016/S01406736(11)60935-5

2. Bork K, Meng G, Staubach P, Hardt J. Hereditary angioedema: new findings concerning symptoms, affected organs, and course. Am J Med. 2006;119(3):267-74 DOI: 10.1016/j.amjmed.2005.09.064

3. Rosen FS, Davis AE III. Deficiencies of $\mathrm{C} 1$ inhibitor. Best Pract Res ClinGastroenterol. 2005;19:251-61 DOI: 10.1016/j.bpg.2004.11.011

4. Csép K, Moldovan D. Genetics of hereditary angioedema. Rev Romana Med Lab. 2010;18(1):7-13

5. Maurer M, Magerl M, Ansotegui I, Aygören-Pürsün E, Betscehl S, Bork K, et al. The international WAO/ EAACI guideline for the management of hereditary angioedema - the 2017 revision and update. Allergy. 2018;73(8):1575-96 DOI: 10.1111/all.13384

6. Germenis AE, Speletas M. Genetics of hereditary angioedema revisited. Clin Rev Allergy Immunol. 2016;51(2):170-82 DOI: 10.1007/s12016-016-8543-x

7. Speletas M, Szilagyi A, Psarros F, Moldovan D, Magerl M, Kompoti M, et al. Hereditary angioedema: molecular and clinical differences among European populations. J Allergy Clin Immunol. 2015;135(2):570-3 DOI: 10.1016/j.jaci.2014.08.007

8. Bygum A, Fagerberg CR, Ponard D, Monnier N, Lunardi J, Drouet C. Mutational spectrum and phenotypes in Danish families with hereditary angioedema because of $\mathrm{C} 1$ inhibitor deficiency. Allergy. 2011;66:76-84 DOI: 10.1111/j.1398-9995.2010.02456.x

9. Rijavec M, Korošec P, Šilar M, Zidarn M, Miljković J, Košnik M. Hereditary Angioedema Nationwide Study in Slovenia Reveals Four Novel Mutations in SERPING1 Gene. PLoS ONE. 2013;8(2):e56712 DOI: 10.1371/journal.pone. 0056712

10. Andrejević S, Korošec P, Šilar M, Košnik M, Mijanović R, Bonači-Nikolić B, et al. Hereditary Angioedema Due to $\mathrm{C} 1$ Inhibitor Deficiency in Serbia: Two Novel
Mutations and Evidence of Genotype- Phenotype Association. PLoS ONE. 2015;10(11):e0142174 DOI: 10.1371/journal.pone.0142174

11. Xu YY, Zhi YX, Yin J, Wang LL, Wen LP, Gu JQ, et al. Mutational spectrum and geno-phenotype correlation in Chinese families with hereditary angioedema. Allergy. 2012;67(11):1430-6 DOI: 10.1111/all.12024

12. Sakshaug JW, West BT. Important considerations when analyzing health survey data collected using a complex sample design. Am J Public Health. 2014;104(1):15-6 DOI: 10.2105/AJPH.2013.301515

13. IBM. SPSS Complex Samples 22. Online: http://www. sussex.ac.uk/its/pdfs/SPSS_Complex_Samples_22.pdf Accessed: 11.01.2019

14. Bouillet L, Launay D, Fain O, Boccon-Gibod I, Laurent $\mathrm{J}$, Martin L, et al. Hereditary angioedema with $\mathrm{C} 1$ inhibitor deficiency: clinical presentation and quality of life of 193 French patients. Ann Allergy Asthma Immunol. 2013;111(4):290-4 DOI: 10.1016/j.anai.2013.07.012

15. Bygum A. Hereditary angio-oedema in Denmark: a nationwide survey. Br J Dermatol. 2009; 161:1153-1158 DOI: 10.1111/j.1365-2133.2009.09366.x

16. Zanichelli A, Arcoleo F, Barca MP, Borrelli P, Bova M, Cancian M, et al. A nationwide survey of hereditary angioedema due to $\mathrm{C} 1$ inhibitor deficiency in Italy. Orphanet J Rare Dis. 2015;10:11 DOI: 10.1186/s13023015-0233-x

17. Speletas M, Szilagyi A, Csuka D, Koutsostathis N, Psarros F, Moldovan D, et al. F12-46C/T polymorphism as modifier of the clinical phenotype of hereditary angioedema. Allergy. 2015;70(12):1661-4 DOI: 10.1111/all.12714

18. Cicardi M, Zingale LC, Zanichelli A, Pappalardo E, Cicardi B. C1 inhibitor: molecular and clinical aspects. Springer Semin Immun. 2005;27:286-98 DOI: 10.1007/s00281-005-0001-4

19. Pappalardo E, Zingale LC, Cicardi M. C1 inhibitor gene expression in patients with hereditary angioedema: quantitative evaluation by means of real-time RTPCR. J Allergy Clin Immunol. 2004;114(3):638-44 DOI: 10.1016/j.jaci.2004.06.021

20. Pappalardo E, Cicardi M, Duponchel C, Carugati A, Choquet S, Agostoni A, et al. Frequent de novo mutations and exon deletions in the $\mathrm{C} 1$ inhibitor gene of patients with angioedema. J Allergy Clin Immunol. 2000;106(6):1147-54 DOI: 10.1067/mai.2000.110471

21. Loules G, Zamanakou M, Parsopoulou F, Vatsiou S, 
Psarros F, Csuka D, et al. Targeted next-generation sequencing for the molecular diagnosis of hereditary angioedema due to C1-inhibitor deficiency. Gene. 2018;667:76-82 DOI: 10.1016/j.gene.2018.05.029

22. Pappalardo E, Caccia S, Suffritti C, Tordai A, Zingale LC, Cicardi M. Mutation screening of $\mathrm{C} 1$ inhibitor gene in 108 unrelated families with hereditary angioedema: Functional and structural correlates. Hum Immunol 2008;45:3536-44 DOI: 10.1016/j.molimm.2008.05.007

23. Lopez-Lera A, Garrido S, Roche O, Lopez-Trascasa M. SERPING1 mutations in 59 families with hereditary angioedema. Mol Immunol 2011;49:18-27 DOI: 10.1016/j.molimm.2011.07.010

24. Stenson PD, Mort M, Ball EV, Shaw K, Phillips A, Cooper DN. The Human Gene Mutation Database: building a comprehensive mutation repository for clinical and molecular genetics, diagnostic testing and personalized genomic medicine. Hum Genet. 2014;133(1):1-9 DOI: 10.1007/s00439-013-1358-4

25. Kalmár L, Hegedüs T, Farkas H, Nagy M, Tordai A. HAEdb: a novel interactive, locus-specific mutation database for the $\mathrm{C} 1$ inhibitor gene. Hum Mutat. 2005;25(1):1-5 DOI: 10.1002/humu.20112

26. Amrani N, Sachs MS, Jacobsen A. Early nonsense: mRNA decay solves a translational problem. Nat Rev Mol Cell Biol. 2006;7(6):415-25 DOI: 10.1038/ nrm1942

27. Carter PE, Duponchel C, Tosi M, Fothergill JE. Complete nucleotide sequence for the $\mathrm{C} 1$ inhibitor with an unusually high density of Alu elements. Eur J Biochem. 1991;197(2):301-8 DOI: $10.1111 / j .1432-1033.1991$. tb15911.x

28. Antonarakis SE. Recommendations for a nomenclature system for human gene mutations. Nomenclature Working Group. Hum Mutat. 1998;11(1):1-3 DOI: 10.1002/(SICI)1098-1004(1998)11:1<1::AID-HUMU1>3.0.CO;2-O

29. Bafunno V, Bova M, Loffredo S, Divella C, Petraroli A, Marone G, et al. Mutational Spectrum of the $\mathrm{C} 1$ Inhibitor Gene in a Cohort of Italian Patients with Hereditary Angioedema: Description of Nine Novel Mutations. Annals of Human Genetics. 2014;78(2):73-82 DOI: 10.1111/ahg. 12052

30. Davies MJ, Lomas DA. The molecular aetiology of the serpinopathies. Int J Biochem Cell Biol .2008;40:127386 DOI: $10.1016 /$ j.biocel.2007.12.017
31. Eldering E, Verpy E, Roem D, Meo T, Tosi M. $\mathrm{COOH}$-terminal substitutions in the serpin $\mathrm{C} 1$ inhibitor that cause loop overinsertion and subsequent multimerization. J Biol Chem. 1995;270:2579-87 DOI: 10.1074/jbc.270.6.2579

32. Aulak KS, Eldering E, Hack CE, Lubbers YP, Harrison RA, Mast A, et al. A hinge region mutation in C1-inhibitor (Ala436 $\rightarrow$ Thr) results in nonsubstrate-like behavior and in polymerization of the molecule. J Biol Chem. 1993;268:18088-94

33. Duponchel C, Djenouhat K, Frémeaux-Bacchi V, Monnier N, Drouet C, Tosi M. Functional analysis of splicing mutations and of an exon 2 polymorphic variant of SERPING1/C1NH. Hum Mutat. 2006;27(3):295-6 DOI: $10.1002 /$ humu.9414

34. Carter PE, Duponchel C, Tosi M, Fothergill JE. Complete nucleotide sequence for the $\mathrm{C} 1$ inhibitor with an unusually high density of Alu elements. Eur J Biochem. 1991;197(2):301-8 DOI: 10.1111/j.1432-1033.1991. tb15911.x

35. Antonarakis SE. Recommendations for a nomenclature system for human gene mutations. Nomenclature Working Group. Hum Mutat. 1998;11(1):1-3 DOI: 10.1002/(SICI) 1098-1004(1998)11:1<1::AID-HUMU1>3.0.CO;2-O

36. Parad RB, Kramer J, Strunk RC, Rosen FS, Davis AE. Dysfunctional C1 inhibitor Ta: deletion of Lys-251 results in acquisition of an N-glycosylation site. Proc Natl Acad Sci U S A 1990;87(17):6786-90 DOI: 10.1073/ pnas.87.17.6786

37. Kramer J, Rosen FS, Colten HR, Rajczy K, Strunk RC. Transinhibition of $\mathrm{C} 1$ inhibitor synthesis in type I hereditary angioneurotic edema. J Clin Invest. 1993;91:1258-62 DOI: 10.1172/JCI116290

38. Ernst SC, Circolo A, Davis AE 3rd, Gheesling-Mullis K, Fliesler M, Strunk RC. Impaired production of both normal and mutant $\mathrm{C} 1$ inhibitor proteins in type I hereditary angioedema with a duplication in exon 8 . J Immunol. 1996;157:405-10

39. Panagiota G, Gedeon L, Maria Z, Maria K, Dorottya Cs, Fotis P, et al. The KLKB1- Ser143Asn polymorphism: a new genetic biomarker predicting the age of disease onset in patients with hereditary angioedema due to C1-INH deficiency (C1-INH-HAE). Allergy Asthma Clin Immunol. 2017;13(2):29 